\title{
A novel optimization approach to forest height reconstruction from multi-baseline data
}

\author{
A. Capozzoli, G. D'Elia, A. Liseno \\ Dipartimento di Ingegneria Elettronica e delle \\ Telecomunicazioni \\ Università di Napoli Federico II \\ via Claudio 21, I 80125 Napoli (Italy) \\ Email: g.delia@unina.it
}

\author{
A. Moreira, K.P. Papathanassiou \\ Deutsches Zentrum für Luft- und Raumfahrt \\ Institut für Hochfrequenztechnik und Radarsysteme \\ D 82234 Wessling (Germany) \\ Email: kostas.papathanassiou@dlr.de
}

\begin{abstract}
The paper deals with the problem of reconstructing the height of forests from polarimetric/multi-baseline SAR data. The approach consists of optimizing an objective functional defined as the distance between the measured data and the data predicted by the model at the actual estimate of the unknowns.

We indicate the role of global optimization on the performance of the forest height reconstruction algorithm. As global optimizer, a multilevel single-linkage method, which incorporates a local optimization into the global search, is exploited, thus offering computational efficiency and reliability. The performance of the method are illustrated against numerically simulated data.
\end{abstract}

Keywords - Forest height reconstruction; Multi-track; Fully polarimetric data.

\section{INTRODUCTION}

Monitoring forested areas has a strong relevance for controlling the health status of the environment and for a continuous control of the biomass dynamics on the climate changes [1]. Indeed, by means of "allometric equations", mapping the height of wide forested areas provides a first estimate of the amount of natural biomass [2].

For this reason, in the last decade, attention has been devoted to the development of remote sensing approaches for recovering forest height profiles from polarimetric interferometric or multi-pass SAR data [3-6].

Forest height reconstruction from multi-pass data may be dealt with by referring to different formulations, depending on the considered dataset. For example, as datum of the problem, the electric field scattered by the scenario of interest may be assumed. Alternatively, the coherency between the different acquired baselines may be used to provide the unknown-to-data mapping. Again, the only-amplitude of the baseline coherency may be employed, by renouncing, as a matter of fact, to the exploitation of the coherency phase which, especially for low coherency regions, may be significantly noisy.

In all the mentioned cases, the problem may be faced by dealing with an (isotropic or anisotropic) scattering model based on a two-layer description of the forest vertical structure, the theoretical field scattered by the scene being considered as the superposition of the independent scattering contributions from the volume and the ground [3-5]. A solution to the forest height estimation, then, can be given by optimizing a cost functional accounting for the distance between the acquired and theoretically predicted data. Furthermore, a constrained optimization can be carried out to account for the available knowledge about the unknowns and/or their intrinsic properties.

A first possibility to perform the optimization is by a gradient-based algorithm, and by introducing a priori information allowing to identify the region of attraction of the solution [7]. A second possibility is to employ a more time consuming algorithm $[8,9]$, but avoiding the stuck into a "false solution".

This paper is focussed on the role of the optimization scheme on the performance achievable in forest height reconstruction.

As known, the high dimensionality of the problem is the main obstacle towards the use of global optimization [8,9]. The amount of computing time required to get a reliable solution, indeed, increases very fast as long as the number of variables increase. Many optimization algorithms have good performances when few variables are involved, but become impractical when dealing many variables, unless heuristic tricks are introduced to speed up convergence $[8,9]$.

To pursue the goal of this paper, attention is here given to the formulation employing, as datum of the problem, the coherency between the acquired baselines. In this framework, first applications of local optimization to airborne experimental data have been performed in [7], with encouraging results. In this paper, we present a numerical analysis showing how, with an appropriate choice of the optimization scheme $[8,9]$, the performance of a forest height reconstruction algorithm may be satisfactory, while keeping low the overall computational requirements and reducing at the same time the amount of needed a priori information on the unknowns.

\section{GLOBAL OPTIMIZATION AND FOREST HEIGHT RECONSTRUCTION}

The relevant geometry is illustrated in Fig. 1.

The observed scenario is assumed to consist of a forested area, whose vertical structure is modelled as two-layers [3,5]. 
The upper layer (volume) has thickness $h_{V}$, contains trunks, branches and leaves, and is regarded as an aggregate of random particles. The lower layer (ground) is dealt with as an impenetrable underlying soil.

The scene is observed from different flight tracks occurring at the same height $h$ and with the same incidence angle $\theta_{i}$, so that $N$ baselines are available for $b=b_{1}, b_{2}, \ldots, b_{N}$, the master being the track at $b=0$.

Each one of the SAR images exhibits an ambiguity along the $s$ direction, which is aimed to be solved by means of the multi-track data. In particular, our aim is to recover the projection of the vegetation thickness along the $s$ direction, namely $h_{V}^{\prime}$, so that the forest height can be subsequently restored from $h_{V}=h^{\prime}{ }_{V} \cos \theta_{i}$.

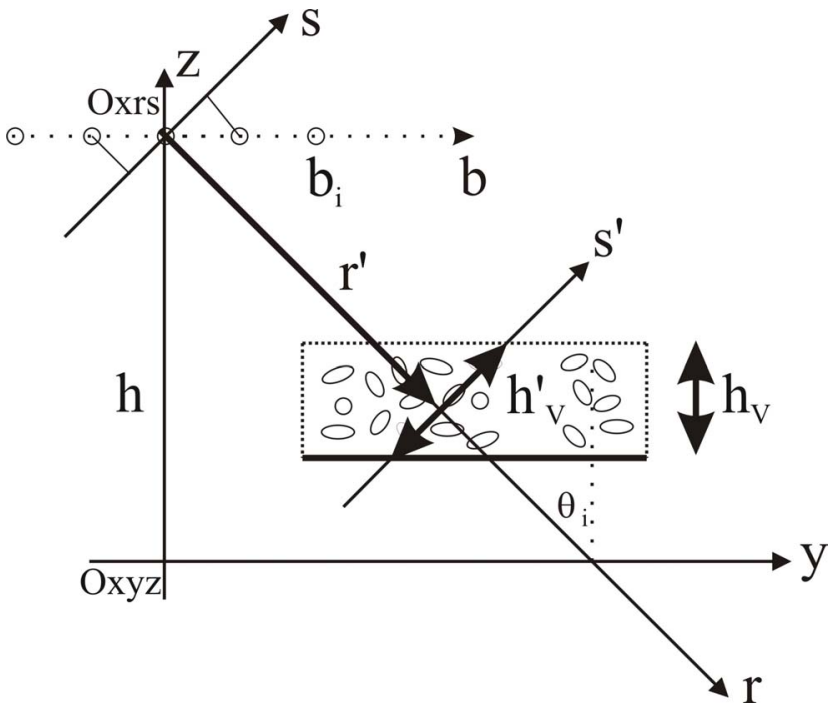

Figure 1. Geometry of the multibaseline forest height reconstruction problem.

To this end, we resort to the use of the interferometric coherency $\gamma^{X Y}$ (b) for the generic polarization XY between the available tracks, which is linked to four unknown real parameters, that is, the forest height $h_{V}$, the underlying ground topography $s_{0}$, the ground-to-volume scattering ratio $\rho_{\Gamma / \chi}^{X Y}$, and the extinction $\sigma$, for a fixed polarization [7]. With the aim of determining the forest height, then, besides $h_{V}$, also the underlying topography, the extinction and the ground-tovolume scattering intensities should be searched for. Nevertheless, by exploiting coherency between the available baselines and by facing a non-linear system of equations, it is possible to obtain a determined system of equations by only 2 single-polarized baselines. The use of any further polarization adds two more real data at the price of one more real unknown ground-to-volume scattering intensity.

In order to retrieve the mentioned unknown parameters, the reconstruction algorithm herein employed performs a minimization of an objective functional accounting for the "distance" between the theoretically predicted coherency $\gamma^{X Y}(b)$ and that $\tilde{\gamma}^{X Y}$ determined from the data.
In the fully-polarized case, the algorithm then consists into the optimization of the functional

$$
\begin{aligned}
& \Phi\left(h_{V}, s_{0}{ }_{0}, \rho_{\Gamma / \chi}^{X_{1} Y_{1}}, \rho_{\Gamma / \chi}^{X_{1} Y_{2}}, \rho_{\Gamma 2}^{X_{2} Y_{2}}, \sigma\right)= \\
& \sum_{\substack{p, q=1 \\
p \neq q}}^{2} \sum_{n=1}^{N}\left|\gamma^{X_{p} Y_{q}}\left(b_{n}\right)-\tilde{\gamma}^{X_{p} Y_{q}}\left(b_{n}\right)\right|^{2}
\end{aligned}
$$

As it can be seen, the reconstruction scheme can flexibly accommodate all the available multi-baseline data and straightforwardly account also for single- or dual-polarized acquisitions, when fully-polarimetric data are not available, by simply discarding corresponding terms in the functional (2).

Moreover, in many instances of practical relevance, a priori information about the observed forested scenario is available. To host such cases, the optimization of $\Phi$ is here worked out as a constrained minimization to explicitly account for such a priori knowledge as well as for the intrinsic properties of the unknowns (e.g., positivity of $h^{\prime}$, and $\rho_{\Gamma / \chi}^{X Y}$ ) [7].

Discrete constraints on the extinction are also enforced, during the optimization procedure, by allowing $\sigma$ to assume a prescribed, discrete and finite set of values, thus achieving a regularization of the behaviour of the reconstruction algorithm.

Finally, the optimization of functional (1) is here performed by means of a global optimization approach. The choice of the global optimizer should be carefully performed, as global optimization algorithms suffer from a significant computational burden $[8,9]$. In this paper, we employ a multilevel singlelinkage method $[8,9]$, which puts together the local and global optimization stages, with appealing numerical and theoretical properties.

\section{NUMERICAL RESULTS}

In this Section, the results obtained against numerically simulated data are described. The results we are going to illustrate aim at assessing, against a numerical test-bed, the feasibility and the potentiality of the approach in simple controlled conditions, namely, in absence of temporal decorrelation, and motion or co-registration errors.

In order to produce synthetic data according to a forward scattering model which is different from that employed for the inversions (illustrated in Section 2), we have simulated the vegetation layer as a discrete set of ellipsoidal particles, randomly located and oriented within a slab of thickness $h_{V}$. Each particle exhibits a random "scattering amplitude" with real and imaginary parts uniformly distributed in $(0,1)$. The scattering amplitude is moreover "damped" due to the extinction of the field during its propagation within the forest slab. In particular, the extinction coefficient has been chosen equal to $0.21 \mathrm{~dB} / \mathrm{m}$, as is realistic for temperate forests [10]. As far as the particle density within the forest slab is concerned, an average number of 10 particles per meter has been assumed. 
For the sake of simplicity, the presented analysis refers to a flat underlying soil, simulated to be impenetrable, with a reflection coefficient so that to obtain a certain desired groundto-volume scattering ratio. To fully account for the polarimetric nature of the radiation which, in general, relevantly helps to improve the accuracy of the reconstructions [5], the data have been generated at three different polarizations, with ground-tovolume scattering ratios of $-5,-10$, and $-15 \mathrm{~dB}$, and corrupted by noise with an overall Signal to Noise (SNR) ratio of $30 \mathrm{~dB}$. Finally, the flight height has been assumed to be $3 \mathrm{~km}$ and a mean incidence angle of $45^{\circ}$ has been considered.

The worked example refers to the case of 3 acquisitions, with $b=0,10,20 \mathrm{~m}$, for a forest height of $25 \mathrm{~m}$, and an underling soil located at $s_{0}=10 \mathrm{~m}$. Positivity constraints have been enforced to $h_{V}$ as well as for the ground-to-volume scattering ratios, whereas no constraint has been considered for the ground topography. Of course, the reconstructed $s_{0}$ will be the wrapped version of the actual underlying soil profile. Moreover, values for $\sigma$ equal to $\left(\begin{array}{llll}0.11 & 0.16 & 0.21 & 0.26\end{array}\right.$ $0.31) \mathrm{dB} / \mathrm{m}$ have been adopted for the discrete regularization of the reconstruction process. Finally, before performing the inversions, the second-order statistics of the field have been estimated by averaging over 49 (i.e., 7x7) pixels.

\section{A.1 Global optimization with a forest of $25 \mathrm{~m}$}

Figs. 2 and 3 show the results achieved by the global optimization algorithm. The reconstructions are henceforth illustrated by a blue, continuous line and the actual values of the reconstructed parameters by a red, dashed line.

\section{A. 2 Local optimization with a forest of $25 \mathrm{~m}$}

Figs. 4 and 5 depict the same outcomes, but now reached by the local optimization [7]. We explicitly note that, by increasing the amount of a priori information, the performance of local optimization improve, as it can be inferred by Fig. 6. In particular, for this case, $h_{V}$ has been enforced to vary within the interval $(0,50) \mathrm{m}$ and $s_{0}$ to vary in $(5,15) \mathrm{m}$.

\section{B. Global optimization with a forest of $40 \mathrm{~m}$}

In order to further stress the capability of the global optimization algorithm to correctly reconstruct a forest height profile, Fig. 7 finally illustrates a test against a $40 \mathrm{~m}$ tall simulated forest.

\section{Computational burden}

To evaluate the feasibility of global optimization in terms of computational burden, Table 1 shows a comparison of the overall computation time required for the reconstruction of the $25 \mathrm{~m}$ tall simulated forest in the local and global cases. The inversions have been performed on an AMD Athlon $643500+$ $2.21 \mathrm{GHz}$ with $2 \mathrm{~Gb}$ of RAM.

\begin{tabular}{|c|c|}
\hline Global optimization & 5 minutes \\
\hline Local optimization & 1 minute \\
\hline
\end{tabular}

Table 1. Computation time for the local and global optimization algorithms.

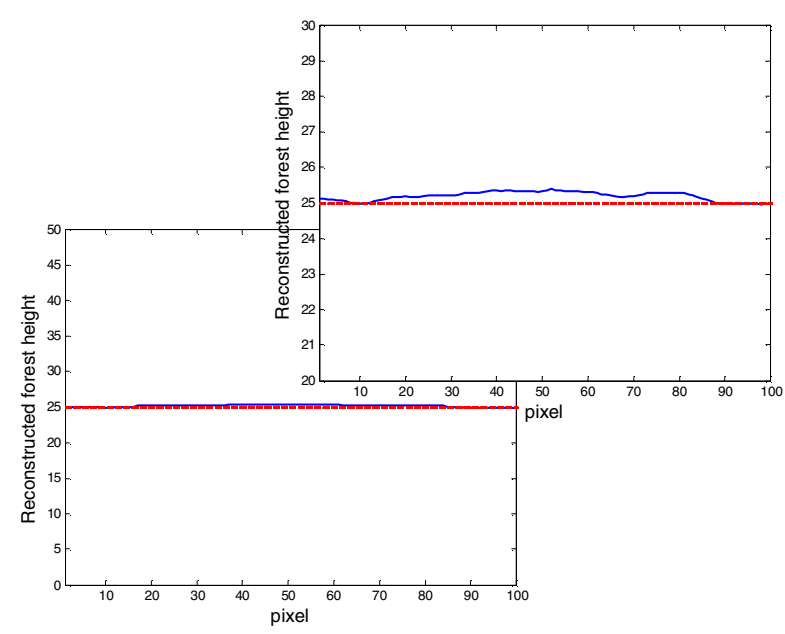

Figure 2. Reconstruction, by global optimization, of a forest height of $25 \mathrm{~m}$.

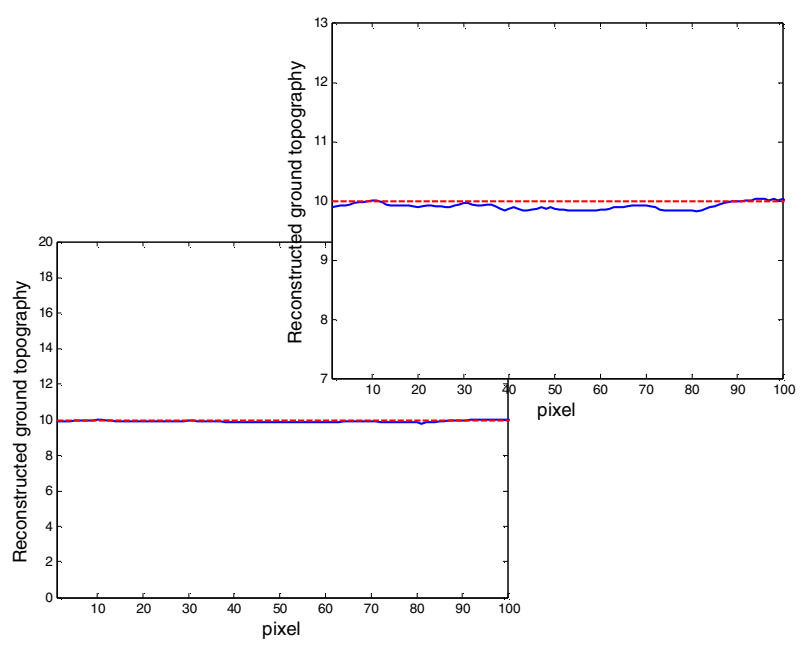

Figure 3. Reconstruction, by global optimization, of a flat ground topography.

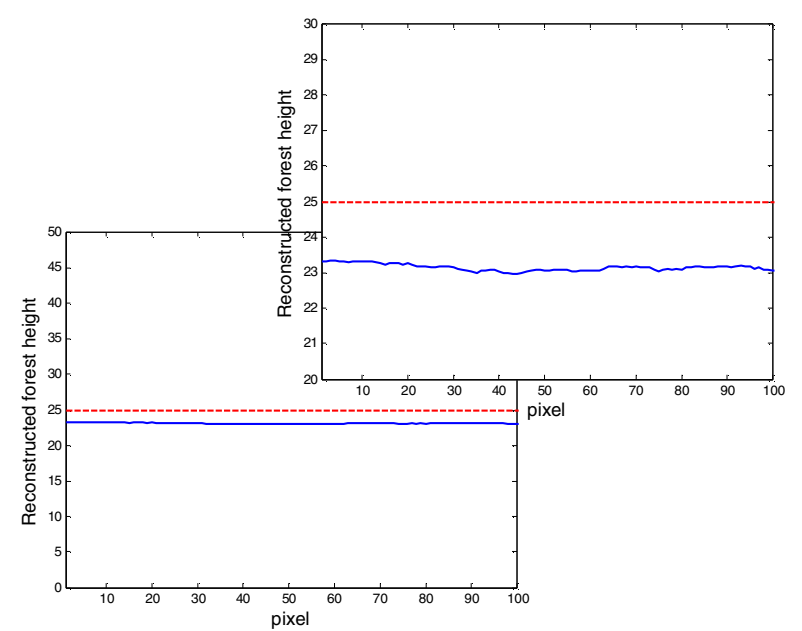

Figure 4. Reconstruction, by local optimization, of a forest height of $25 \mathrm{~m}$. 


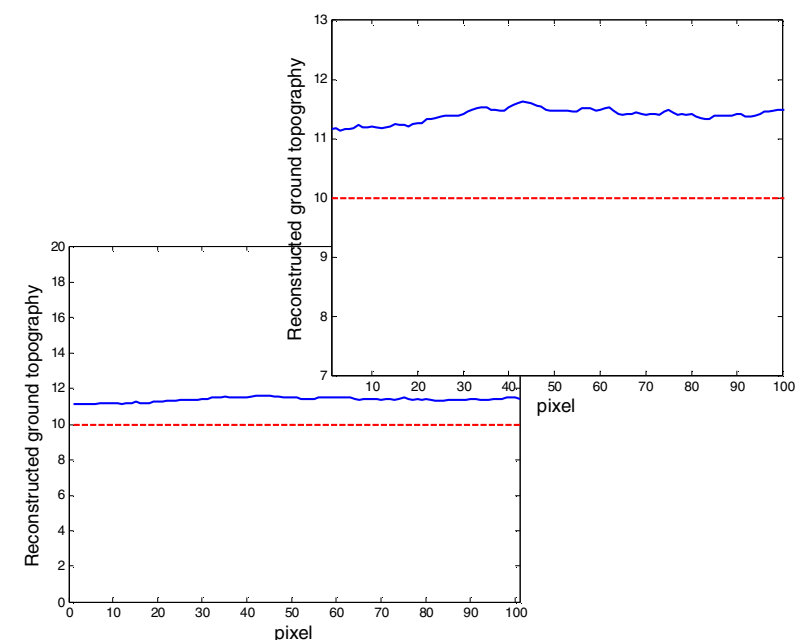

Figure 5. Reconstruction, by local optimization, of a flat ground topography.

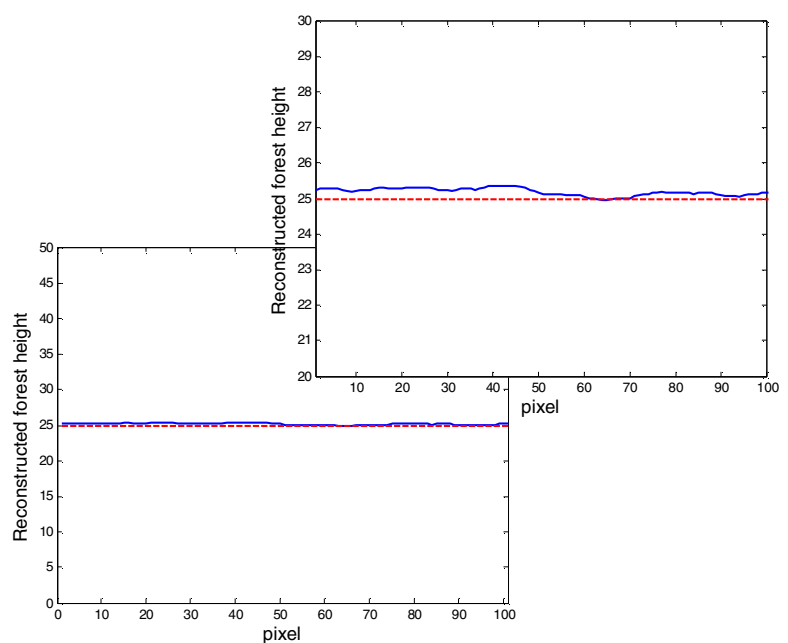

Figure 6. Reconstruction, by local optimization, of a forest height profile of $25 \mathrm{~m}$ by narrowing the constraints.

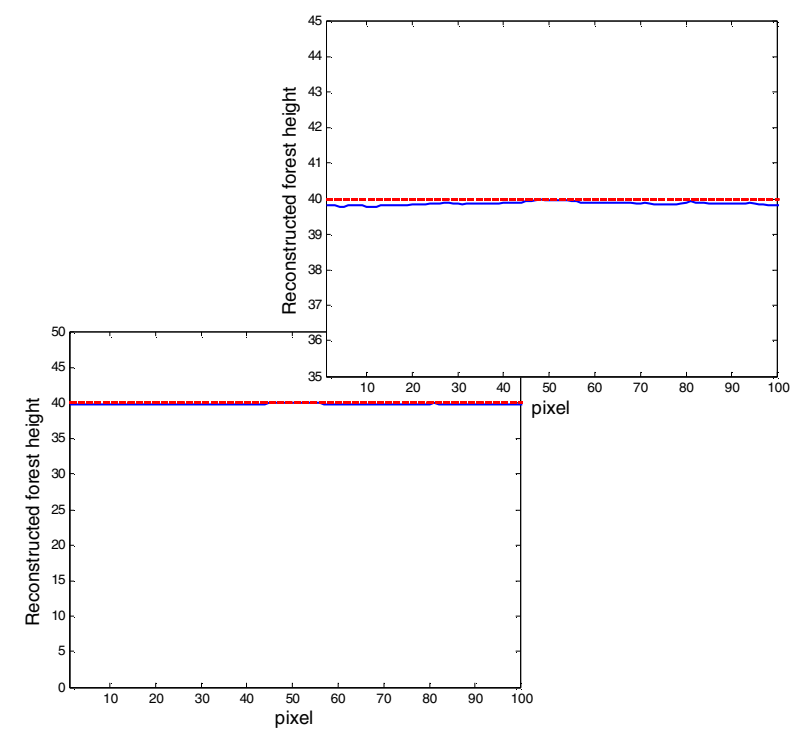

Figure 7. Reconstruction, by global optimization, of a forest height profile of $40 \mathrm{~m}$.

\section{CONCLUSIONS AND FUTURE DEVELOPMENTS}

In this paper, we have discussed the feasibility and potentiality of an algorithm for the reconstruction of forest height profiles from polarimetric/multi-track SAR data. The algorithm exploits a "smart" optimization scheme of the multistart class, i.e. a multilevel single-linkage approach.

The comparison to local optimization has shown the usefulness of innovative global optimizers in the framework of the forest height reconstruction problem. It should be noticed that the employed global algorithm can be easily parallelized, thus further reducing the needed computation time and resulting to be very appealing thanks to the recent wide spreading of dual, or multi, processor computers.

Future developments of this work will regard the testing of the reconstruction procedure to more complex scenarios, such as a non-flat underlying ground, and the analysis of the effect of the parameters of the acquisition configuration (such as, baseline length, number of acquisitions) on the accuracy of the reconstructions. Furthermore, the properties of the onlyamplitude formulation of the coherence and the role of polarization diversity (i.e., single-pol or dual-pol inversions) will be explored.

\section{REFERENCES}

[1] R.N. Treuhaft, B.E. Law, G.P. Asner, "Forest attributes from radar interferometric structure and its fusion with optical remote sensing," BioScience, vol. 54, n. 6, pp. 561-571, Jun. 2004.

[2] T. Mette, K. Papathanassiou, I. Hajnsek, H. Pretzsch, P. Biber, "Applying a common allometric equation to convert forest height from Pol-InSAR data to forest biomass", Proc. of the IEEE Geosci. Remote Sens. Soc., Anchorage, USA, 2004, pp. 272-275.

[3] R.N. Treuhaft, S.N. Madsen, M. Moghaddam, J.J. van Zyl, "Vegetation characteristics and underlying topography from interferometric radar", Radio Sci., vol. 31, n. 6, pp. 1449-1486, 1996.

[4] R.N. Treuhaft, P.R. Siqueira, "Vertical structure of vegetated land surfaces from interferometric and polarimetric data", Radio Sci., vol. 35, n. 1, pp. 141-178, Jan. 2000.

[5] S.R. Cloude, K.P. Papathanassiou, "Three-stage inversion process for polarimetric SAR interferometry", IEE Proc. - Radar, Sonar, Nav., vol. 150 , n. 3, pp. 125-134, Jun. 2003.

[6] S.R. Cloude, M.L. Williams, "A coherent EM scattering model for dual baseline PolInSAR", Proc. of the IEEE Geosci. Remote Sens. Soc., Toulouse, France, Jul. 21-25 2003, pp. 1423-1425.

[7] A. Capozzoli, G. D’Elia, A. Liseno, A. Marino, A. Moreira, K.P. Papathanassiou, "Forest height reconstruction by a multibaseline approach: experimental results", Proc. of the $3^{\text {rd }}$ Int. Workshop on Sci. and Appl. of SAR Polarimetry and Polarimetric Interferometry, Frascati, Italy, Jan. 22-26, 2006.

[8] A. Capozzoli, G. D'Elia, “Global optimization and antennas synthesis and diagnostics, part one: concepts, tools, strategies and performances", Progr. Electromagn. Res. PIER, vol. 56, pp. 195-232, 2006.

[9] A. Capozzoli, G. D'Elia, "Global optimization and antennas synthesis and diagnosis, part two: applications to advanced reflector antennas synthesis and diagnosis techniques", Progr. Electromagn. Res. PIER, vol. 56, pp. 233-261, 2006.

[10] M.L. Williams, "Simulating low frequency SAR clutter from a pine forest", Proc. of the Europ. Conf. on Synthetic Aperture Radar, Munich, Germany, May 23-25, 2000, pp. 149-152. 